\title{
Assay signal as an alternative to titer for assessment of magnitude of an antidrug antibody response
}

\author{
Marta Starcevic Manning ${ }^{*} 1,3$, Mark A Kroenke ${ }^{2}$, Stephanie A Lee ${ }^{1}$, Simon E Harrison ${ }^{1}$, \\ Sarah A Hoofring ${ }^{1}$, Daniel T Mytych ${ }^{2}$ \& Vibha Jawa ${ }^{2,4}$ \\ ${ }^{1}$ Pharmacokinetics \& Drug Metabolism, Amgen, Thousand Oaks, CA 91320, USA \\ ${ }^{2}$ Clinical Immunology, Amgen, Thousand Oaks, CA 91320, USA \\ ${ }^{3}$ One Amgen Center Drive, Mail Stop 30E-3-B, Thousand Oaks, CA 91320, USA \\ ${ }^{4}$ Currently Biologics \& Vaccine Bioanalytics, Merck, Kenilworth, NJ 07033, USA \\ * Author for correspondence: Tel.: +1 805447 4987; mstarcev@amgen.com
}

Background: Titer methods are commonly used to characterize the magnitude of an antidrug antibody response. Assay $\mathrm{S} / \mathrm{N}$ is an appealing alternative, but the circumstances under which use of signal-to-noise $(\mathrm{S} / \mathrm{N})$ is appropriate have not been well defined. Results: We validated both titer and S/N-based methods for several therapeutics. $\mathrm{S} / \mathrm{N}$ correlated strongly with titer both in aggregate and when examined on a per subject basis. Analysis of impact of antibody magnitude on pharmacokinetics yielded the same result using either method. Each assay demonstrated excellent precision, good linearity, and adequate drug tolerance. Conclusion: Under these circumstances, assay $\mathrm{S} / \mathrm{N}$ is a valid alternative to titer for assessment of the magnitude of an antidrug antibody response.

First draft submitted: 24 August 2017; Accepted for publication: 22 September 2017; Published online: 12 October 2017

Keywords: antidrug antibody $\bullet$ immunogenicity $\bullet \mathrm{S} / \mathrm{N} \bullet$ titer

\section{Key terms}

- Immunogenicity: the ability of an antigen to induce an adaptive immune response after administration to humans or animals.

- Antidrug antibody: an antibody elicited by administration of a protein therapeutic which is specific for the therapeutic.

- Antidrug antibody magnitude: a surrogate measure of the concentration of antidrug antibodies detected in patient samples.

- Signal-to-noise $(\mathrm{S} / \mathrm{N})$ : assay response generated by the sample or positive control divided by the response generated by the negative control analyzed on the same plate.

- Titer: the reciprocal of the highest sample dilution that still gives a positive result in the assay, or the reciprocal of the dilution value derived from interpolation from the sample dilution curve at a predetermined cut point

The assessment of antidrug antibody (ADA) responses in clinical samples is a regulatory expectation, and an important component of the safety evaluation of a new therapeutic. ADAs can have a wide variety of consequences ranging from complete neutralization of drug to enhanced exposure, and for this reason it is important to carefully assess how ADA may impact pharmacokinetics (PK), safety, and efficacy [1]. A variety of ADA detection platforms and formats have been explored and the bridging electrochemiluminescence (ECL)-based approach is the most commonly used, especially for monoclonal antibody therapeutics [2,3]. Historically, ADA assays have been purely qualitative, with a positive or negative result reported based on statistically derived assay cut points. However, there are cases where it is important to provide some measure of the magnitude of the immune response. The availability of quasi-quantitative ADA data enables stratification by antibody levels prior to analysis of impact [4]. This ensures that a potential impact of ADA in a small subset of subjects with a robust antibody response is 
not overwhelmed and lost when analyzed with a larger number of ADA positive subjects who exhibit less robust responses. Furthermore, for biosimilar drug development, it is important to demonstrate that the biosimilar has a similar incidence of immunogenicity and that those antibody responses are similar in magnitude relative to the innovator [5,6]. Finally, ADA magnitude can provide meaningful information on the strength and progression of an ADA response across study time points [1]. This is especially useful for evaluating the potential boosting of a pre-existing antibody response following therapeutic administration $[7,8]$. For these reasons, current regulatory guidance recommends further characterization of ADA responses based on magnitude $[9,10]$.

The most commonly used methods for quasi-quantitation of ADA report magnitude as mass units or titer [11]. The determination of mass units is based on the interpolation of sample signal from a positive control dose-response curve. This approach is problematic due to the absence of a reference standard that matches the diversity of the immune response in the clinic [12]. For instance, polyclonal positive controls are typically generated in rabbits. The composition of rabbit antibodies in this preparation may be dramatically different from the study population in terms of epitope specificity, affinity, and antibody isotype and subclass. The more accepted approach, titer, is defined as the reciprocal of the highest sample dilution that still gives a positive result in the assay, or the reciprocal of the dilution value derived from interpolation from the sample dilution curve at a predetermined cut point [1].

Titration is currently the favored method for quasi-quantitation of ADA for several reasons. It is generally understood and accepted by the medical community. No reference standard is required. In addition, dilution of samples may alleviate the interference caused by therapeutic present in the sample. However, titer methods do have some disadvantages. Depending on the dilution scheme and whether interpolation is used to determine the final result, titer values may lack accuracy [1]. Titration assays also cannot differentiate well between low-level ADA responses. If the first dilution produces a negative result, low-level responses are reported with the same titer value equivalent to the assay's minimum required dilution. Finally, titration assays have significant sample volume and resource utilization drawbacks. Analysis of each positive sample involves multiple dilutions, requiring additional sample volume in addition to what is already required for the initial screening/confirmatory assays and the neutralizing antibody assay. Additional analyst time and increased reagent consumption are required to perform a third tier of analysis where each positive sample is converted into six to eight additional samples.

A potential alternative approach for assessing the magnitude of an ADA response is to utilize the assay signal or signal-to-noise $(\mathrm{S} / \mathrm{N})$ value derived from the untreated sample analyzed in the confirmatory assay. $\mathrm{S} / \mathrm{N}$ is defined as the ratio of the sample response divided by the negative control (or background) response. This value is generated for all samples tested in the binding ADA assay and provides a measure of magnitude without retesting the sample in an additional assay. In this study, $\mathrm{S} / \mathrm{N}$ and titer values from three monoclonal antibody therapeutic clinical studies were used to evaluate whether $\mathrm{S} / \mathrm{N}$ is a viable alternative to titer in the assessment of the magnitude of the antidrug antibody response.

\section{Experimental \\ Materials}

Three therapeutic antibodies (referred to as mAb1, mAb2, and mAb3) manufactured by Amgen, Inc. (CA, USA) as high concentration solutions with concentrations ranging from 30 to $100 \mathrm{mg} / \mathrm{ml}$ were used in this study. Stock therapeutic solutions were kept at $-80^{\circ} \mathrm{C}$ and thawed at ambient room temperature prior to use. The positive controls for each ADA method were polyclonal anti-CDR specific antibodies generated in rabbits. Rabbits were immunized with whole $\mathrm{mAb}$ therapeutic and the antisera affinity-purified using the $\mathrm{mAb}$ covalently conjugated to cyanogen bromide-activated Sepharose 4B (GE Healthcare, PA, USA) and subsequent adsorption against human immunoglobulin bound to Sepharose 4B. The capture and detection reagents for the ADA assays were generated by conjugating the monoclonal antibody therapeutics to biotin (Sulfo-NHS-LC-Biotin, Thermo Scientific, MA, USA) or ruthenium (Sulfo-TAG-NHS ester, MSD, MD, USA) according to the manufacturers' instructions. Prior to assay validation, conjugated reagents were characterized to ensure they maintained immunological reactivity after labeling with biotin or ruthenium.

\section{ADA sample collection}

Clinical serum samples were obtained from multiple-dose Phase II studies. For mAb1, serum samples were collected from subjects who were dosed every 1 or 3 months for 24 months with subcutaneous injections. ADA samples were collected prior to dosing at baseline and months 3, 6, 9, 12, 13, 15, 18, 21, 24, and 27, 36, 37, 39, 42, 45, 
Table 1. Assay parameters.

\begin{tabular}{|c|c|c|c|c|}
\hline Product & Screening assay cut point & Titer cut point & $\begin{array}{l}\text { Screening assay sensitivity } \\
(\mathrm{ng} / \mathrm{ml})\end{array}$ & $\begin{array}{l}\text { Drug tolerance at } 500 \mathrm{ng} / \mathrm{ml} \text { of } \\
\text { positive control ADA }(\mu \mathrm{g} / \mathrm{ml})\end{array}$ \\
\hline mAb1 & 1.13 & 1.61 & 5.0 & 100 \\
\hline mAb2 & 1.13 & 1.48 & 9.2 & 200 \\
\hline mAb3 & 1.17 & 1.75 & 34.3 & 108 \\
\hline
\end{tabular}

48, and 51. PK samples were collected at baseline, D1 plus 1 week, and months 1, 2, 3, 6, 9, 12, 12 plus 1 week, $13,14,15,18,21,24$, and $27,36,37,38,42,45$, and 48 .

For mAb2, serum samples were collected from subjects who were dosed every 4 weeks with subcutaneous injections. ADA samples were collected prior to dosing at baseline and at weeks 2, 4, 8, 12, 36, and 52.

For mAb3, samples were collected from subjects who received weekly subcutaneous injections of mAb3 for 12 weeks. Serum samples for the detection of anti-mAb3 antibodies were collected prior to dosing at baseline and at weeks $4,8,12$, and 16 .

\section{ADA titer samples}

For mAb1, all available ADA positive samples within a single study were tested in the validated titer method. For $\mathrm{mAb} 2$, all available ADA samples from subjects with at least one positive ADA time point collected up to a certain data cutoff date were analyzed in the validated titer method. For mAb3, reagent limitations precluded the titer evaluation of all ADA positive samples. Therefore, samples for titer evaluations were selected to ensure that all types of antibody responses (i.e., pre-existing/developing, persistent/transient, and neutralizing/non-neutralizing) were reflected in the sample subset. Titer and $\mathrm{S} / \mathrm{N}$ results were generated for 81 subjects (348 samples) for $\mathrm{mAb} 1,22$ subjects (97 samples) for mAb2, and 31 subjects (105 samples) for mAb3.

\section{ADA bridging assays}

Validated ECL-based bridging assays were used to detect the presence of binding ADAs against mAb1, mAb2, and mAb3. A similar assay format was used for all three therapeutics and was described previously [4].

Briefly, control and study serum samples were acid treated to enhance detection in the presence of excess therapeutic. Samples were incubated with a mixture of biotinylated and ruthenylated therapeutic in neutralizing buffer. The neutralized samples were transferred to wells of a blocked avidin or streptavidin coated plate to immobilize bridged complexes. The plate was washed to remove any unbound complexes and read buffer containing tripropylamine was added to each well. Using a Meso Scale Discovery Sector Imager 6000 instrument (MSD, MD, USA), an electrical current was conducted across the electrodes on the plate resulting in a series of oxidationreduction reactions. The ECL signal produced by the interaction between the ruthenium (from the captured complex) and tripropylamine was measured and quantified as ECL units.

The $\mathrm{S} / \mathrm{N}$ of the ADA responses was calculated by dividing the raw mean ECL signal from the study sample by the mean signal derived from the negative control (pooled normal human serum) analyzed on the same plate. The screening assay S/N cut point was calculated using a 95\% one-sided prediction interval based on healthy donor serum. Samples exceeding this cut point were further tested in the confirmatory immunoassay by depletion with excess unlabeled therapeutic. The confirmatory assay cut point was established using a one sided $99.8 \%$ prediction interval for $\mathrm{mAb} 1$ and a $99 \%$ prediction interval for $\mathrm{mAb} 2$. The $\mathrm{mAb} 3$ assay was validated prior to current cut point guidance, and the confirmatory assay cut point was set at $\% \mathrm{~S} / \mathrm{N}$ reduction of 50 . Samples with a $\mathrm{S} / \mathrm{N}$ value exceeding the screening assay cut point and a percent depletion value exceeding the confirmatory assay cut point were deemed positive. Assay parameters such as assay cut point, sensitivity, and drug tolerance are listed in Table 1.

\section{ADA titer assays}

Samples and controls were titered in duplicate in pooled normal human serum using a 1:3 dilution scheme. To account for the 1:20 minimum required dilution in the assay, titer values were multiplied by 20 to obtain a titer range of $1: 20,1: 60,1: 180,1: 540,1: 1620$, and 1:4860. These diluted samples were analyzed via the bridging assay described above.

In the titer method, linear interpolation at the titer cut point (TCP) was used to calculate the antibody titer value from sample dilutions producing a $\mathrm{S} / \mathrm{N}$ value above and below the TCP. As the screening assay cut point was 
located within the lower plateau of the assay, the TCP was established for each mAb therapeutic using the response variation of naive healthy donors (mean $+6 \mathrm{SDs}$ ) from the bridging assay validation.

The final titer value, based on the mean of duplicate samples, was reported for any samples that had a $\mathrm{S} / \mathrm{N}$ result above the TCP at a dilution of $>1: 20$. The titer of samples testing below the TCP at the minimum required dilution or above the TCP at the highest dilution were reported as $<20$ or $>4860$, respectively. Titer assay acceptance criteria required that sample duplicates used in the titer calculation must have a CV $(\%) \leq 20$ and that the positive control titer must be between the acceptable titer range validated for each therapeutic.

A comparison of drug tolerance between the titer and $\mathrm{S} / \mathrm{N}$ methods was performed during the titer assay validation for each drug product. Samples containing various concentrations of positive control antibody $(0.05$, 0.1 , and $0.5 \mu \mathrm{g} / \mathrm{ml})$ and various amounts of therapeutic $(0,0.5,5$, and $50 \mu \mathrm{g} / \mathrm{ml})$ were prepared and analyzed. The sample containing only antibody was compared with the sample containing the same concentration of antibody in the presence of therapeutic. The \% recovery was calculated using the following formula:

Average Titer or S/N (antibody + therapeutic sample) ${ }^{*} 100 /$ Average Titer or S/N (antibody only sample)

\section{mAb1 drug concentration assay}

Free $\mathrm{mAb} 1$ concentrations were measured using a validated colorimetric assay. Calibration standards and quality controls (QC) were prepared by spiking mAb1 into 100\% human serum. Standard, QC, blank and study samples were added to a plate that had been passively coated with a mouse antihuman mAb1 monoclonal antibody (Amgen, Inc.). After capture of mAb1 to the immobilized antibody, unbound materials were removed by a wash step. Horseradish peroxidase conjugated to another mouse anti-mAb1 monoclonal antibody (Amgen, Inc.) was added for detection of captured mAb1. After another wash step, a tetramethylbenzidine peroxide substrate solution (Seracare Life Sciences, MA, USA) was added to produce a colorimetric signal, which was proportional to the amount of $\mathrm{mAb} 1$ bound by the capture reagent. The color development was stopped by the addition of $\mathrm{H}_{2} \mathrm{SO}_{4}$ and the instrument response was measured at $450 \mathrm{~nm}$ with reference to $650 \mathrm{~nm}$. The conversion of optical density units for QC and study samples to concentration was achieved through a computer software mediated comparison to a calibration curve analyzed on the same plate which was regressed according to a four-parameter regression model without weighing.

\section{Statistical analysis}

Spearman's correlation coefficient (rho) was utilized to measure the strength of the association between logtransformed $\mathrm{S} / \mathrm{N}$ and titer values. Statistical significance of the Spearman's correlation coefficient was assessed using a two-tailed p-value. Spearman's correlation coefficient can range from - 1 to 1 where a value of 1 indicates a perfect association of ranks and a value between 0 and 1 indicates that the two variables tested increase or decrease together. Samples producing a titer value of $<20$ (i.e., those that tested below the TCP in the first dilution) or $>4860$ were excluded from the analysis.

$\mathrm{r}^{2}$ regression analysis was used to assess linearity of immunoassay standard curves. An $\mathrm{r}^{2}$ value of 1.0 indicates perfect linearity.

\section{Results \& discussion}

Clinical immunogenicity

Each ADA assay was validated per regulatory guidance and best practices described in industry white papers available at the time of assay validation [13-15]. Screening assay sensitivity was calculated based on a polyclonal rabbit positive control antibody and ranged from 5.0 to $34.3 \mathrm{ng} / \mathrm{ml}$. All three assays were able to detect $500 \mathrm{ng} / \mathrm{ml}$ of polyclonal rabbit positive control ADA in the presence of a minimum of $100 \mu \mathrm{g} / \mathrm{ml}$ of excess drug (Table 1). Good assay precision, linearity within the range of study sample responses, and acceptable impact of drug on $\mathrm{S} / \mathrm{N}$ recovery were considered to be critical for the use of assay signal as a measure of ADA magnitude. Therefore, these parameters were evaluated for all three assays.

Each clinical program utilized a tiered immunogenicity testing strategy. All antibody samples were first tested in a screening $\mathrm{ADA}$ assay and if the $\mathrm{S} / \mathrm{N}$ was above the screening assay cut point, the sample was further tested in a confirmatory assay which involved depletion of signal with unlabeled therapeutic. If a sample tested positive in the confirmatory assay, then the sample was also tested in an in vitro neutralizing antibody assay.

The overall incidence of anti-mAb1 binding antibodies as of month 24 was 60/255 (23.5\%), with 8 subjects (3.1\%) testing positive for in vitro neutralizing antibodies at one or more time points. A subset of study subjects 


\begin{tabular}{|c|c|c|c|c|c|c|}
\hline & \multicolumn{2}{|c|}{ mAb1 } & \multicolumn{2}{|c|}{ mAb2 } & \multicolumn{2}{|c|}{ mAb3 } \\
\hline & $\%$ CV & $n$ & $\%$ CV & $n$ & $\%$ CV & $n$ \\
\hline LPC & $7 \%$ & 28 & $4 \%$ & 10 & $4 \%$ & 6 \\
\hline HPC & $14 \%$ & 81 & $10 \%$ & 31 & $7 \%$ & 6 \\
\hline
\end{tabular}

HPC: High positive control; LPC: Low positive control; mAb: Monoclonal antibody; n: number of independent assay.

\begin{tabular}{|c|c|c|c|c|c|}
\hline & \multicolumn{2}{|c|}{$\mathrm{ADA}^{+}$sample $\mathrm{S} / \mathrm{N}$ range } & \multicolumn{2}{|c|}{ Validation dose response curve $\mathrm{S} / \mathrm{N}$ range } & \multirow[t]{2}{*}{ Dose response curve $r^{2}$} \\
\hline & Low & High & Low & High & \\
\hline mAb1 & 1.22 & 1869.65 & 1.35 & 4638.54 & 0.9993 \\
\hline mAb2 & 1.43 & 398.37 & 1.28 & 620.84 & 0.9884 \\
\hline mAb3 & 1.12 & 51.01 & 1.06 & 77.94 & 0.9861 \\
\hline
\end{tabular}

was also treated month 36 through month 48 and had a cumulative incidence of binding anti-mAb1 antibodies of $19 / 167$ (11.4\%), with 1 subject $(0.6 \%)$ positive for in vitro neutralizing antibodies. During the double-blind phase of the mAb2 study, the overall incidence of anti-mAb2 binding antibodies was 33/317 (10.4\%), with 9 subjects $(2.8 \%)$ showing in vitro neutralizing activity. The $\mathrm{mAb} 3$ clinical study had an overall incidence of anti-mAb3 binding antibodies of $110 / 218$ (50.5\%), with 17/218 (7.8\%) showing in vitro neutralizing activity.

\section{Evaluation of critical characteristics of each ADA detection method}

ADA samples collected at different time points from the same subject may be analyzed on different days. Therefore, $\mathrm{S} / \mathrm{N}$ must demonstrate good assay precision in order to enable the monitoring of immune response progression over time. Inter-assay precision of all three assays was evaluated during validation for the low and high positive controls (LPC and HPC, respectively). The CV ranged from 4 to $7 \%$ for the LPC and from 7 to $14 \%$ for the HPC (Table 2).

The linearity of each assay was also assessed because if $\mathrm{S} / \mathrm{N}$ values from study samples fall within the lower or upper plateaus of the dose response curve, an accurate assessment of ADA magnitude cannot be made. An $\mathrm{r}^{2}$ value was derived from the validation dose response curve covering the range of $S / N$ values observed in each clinical study. All three assays were linear with $\mathrm{r}^{2}$ values ranging from 0.9861 to 0.9993 (Table 3).

\section{Impact of circulating drug on $\mathrm{S} / \mathrm{N}$ versus titer methods}

One potential advantage of a titer based method is that titration dilutes out circulating unlabeled drug, which may result in improved detection of ADAs. In order to understand the importance of the dilution component of titer methods, pooled normal human serum was spiked with various concentrations of rabbit polyclonal ADA along with excess concentrations of the corresponding therapeutic. Both $\mathrm{S} / \mathrm{N}$ and titer values were generated for these samples, and percent signal recovery was calculated by comparing the measured response for samples with various levels of excess drug to those with no drug. At low levels of drug $(0.5$ and $5 \mu \mathrm{g} / \mathrm{ml})$, the percent recovery for $S / \mathrm{N}$ and titer were similar (Table 4). However, at high concentrations of drug $(50 \mu \mathrm{g} / \mathrm{ml})$, there was more impact on $\mathrm{S} / \mathrm{N}$ values compared with titer.

In this experiment, a $50 \mu \mathrm{g} / \mathrm{ml}$ drug concentration was selected in order to robustly test the hypothesis that titer would tolerate high drug levels better than $\mathrm{S} / \mathrm{N}$. While this proved to be true, this concentration represents an extreme case. In postdose ADA samples analyzed for $\mathrm{S} / \mathrm{N}$ and titer within the three clinical studies, the mean drug concentration for $\mathrm{mAb} 1, \mathrm{mAb} 2$, and $\mathrm{mAb} 3$ was $2.1,1.2$, and $1.2 \mu \mathrm{g} / \mathrm{ml}$, respectively. Drug levels within $90 \%$ of the samples were below 7.2, 4.2, and $3.2 \mu \mathrm{g} / \mathrm{ml}$ for $\mathrm{mAb} 1, \mathrm{mAb} 2$, and $\mathrm{mAb} 3$, respectively, and did not exceed $15 \mu \mathrm{g} / \mathrm{ml}$ in any of the samples tested. Therefore, $\mathrm{S} / \mathrm{N}$ values in these three assays demonstrated acceptable tolerance to drug levels present in postdose study samples.

\section{Comparison of S/N \& titer based assessments}

In order to evaluate the relationship between titer and $S / N, \log S / N$ and $\log$ titer values were plotted for each monoclonal antibody (Figure 1). The data were not normally distributed so a Spearman's rank correlation test 


\begin{tabular}{|c|c|c|c|c|c|c|}
\hline Product & ADA $(\mu \mathrm{g} / \mathrm{ml})$ & $\operatorname{Drug}(\mu \mathrm{g} / \mathrm{ml})$ & Titer & $\%$ Titer recovery & $S / N$ & $\% \mathrm{~S} / \mathrm{N}$ recovery \\
\hline \multirow[t]{5}{*}{ mAb1 } & 0.05 & 0 & 160 & - & 5.23 & - \\
\hline & & 5 & 149 & 93 & 4.09 & 78 \\
\hline & 0.5 & 0 & 1796 & - & 47.57 & - \\
\hline & & 0.5 & 1730 & 96 & 39.50 & 83 \\
\hline & & 50 & 1512 & 84 & 11.60 & 24 \\
\hline \multirow[t]{4}{*}{ mAb2 } & 0.1 & 0 & 93.2 & - & 2.86 & - \\
\hline & & 0.5 & 71.9 & 77 & 2.57 & 90 \\
\hline & & 5 & 59.4 & 64 & 2.14 & 75 \\
\hline & 0.5 & 0 & 545.6 & - & 11.40 & - \\
\hline
\end{tabular}

was used to generate a correlation coefficient (rho) and associated two-tailed p-value. For all three monoclonal antibodies, a statistically significant correlation was observed between $\mathrm{S} / \mathrm{N}$ values and titer, with Spearman's rho values in a tight range from 0.9910 to 0.9941 and p-values of $<0.0001$. This data indicates that for this set of monoclonal antibody studies and these specific assays, titer and $\mathrm{S} / \mathrm{N}$ are interchangeable measures of ADA magnitude. For these studies, any analysis of impact of ADA on PK, safety, or efficacy should have the same outcome regardless of which measure of magnitude is used.

To ensure that this correlation was consistently maintained on a per subject basis over the course of a clinical study, individual subject time courses utilizing either titer or $\mathrm{S} / \mathrm{N}$ values were plotted. The subject profiles with at least three available time points and the largest $\mathrm{S} / \mathrm{N}$ values within the titerable range from each study are shown in Figure 2, and are representative of all subject profiles examined. For each product, the kinetics of the ADA response are similar whether titer or $\mathrm{S} / \mathrm{N}$ values are used, indicating that for these studies, $\mathrm{S} / \mathrm{N}$ can be used to monitor progression of the immune response over time.

\section{Impact of ADA on PK}

The ultimate goal of immunogenicity testing is to determine the impact of ADAs. In order to confirm that impact on $\mathrm{PK}$ was detected equivalently with both titer and $\mathrm{S} / \mathrm{N}$ based assessments of magnitude, drug concentrations were plotted against each ADA magnitude measure for the highest dose group in the mAb1 study (Figure 3). The highest dose group was selected because it is most likely to exhibit differentiation between $S / N$ and titer as a result of excess circulating drug which would be diluted only by the titer method. Even in this scenario, high magnitude ADA responses were associated with lower drug levels, regardless of whether $\mathrm{S} / \mathrm{N}$ or titer was used.

\section{Conclusion}

The data presented above indicates that under the appropriate circumstances, assay signal or $\mathrm{S} / \mathrm{N}$ can be used in lieu of titer to assess the magnitude of an $\mathrm{ADA}$ response. In order to use $\mathrm{S} / \mathrm{N}$, the $\mathrm{ADA}$ assay must meet several important requirements. First, it must exhibit reasonable linearity in a range of expected $\mathrm{S} / \mathrm{N}$ values during clinical sample analysis. This should be evaluated during validation and confirmed after the clinical data is available to ensure the validation data covers the full range of observed values. If study sample results fall within the upper plateau or hook effect region of the assay, $\mathrm{S} / \mathrm{N}$ values would be inaccurate. Furthermore, $\mathrm{S} / \mathrm{N}$ values must demonstrate acceptable inter-assay precision in order to enable accurate monitoring of potential fluctuations of the ADA response. Finally, the assay must have sufficient drug tolerance. Even levels of drug which do not impact the qualitative outcome of the assay (positive/negative) could impact $\mathrm{S} / \mathrm{N}$ values. This is potentially a confounding factor when assessing how ADA magnitude impacts PK, safety, and efficacy. Consequently, close attention should be paid to how excess drug impacts $\mathrm{S} / \mathrm{N}$ values during assay validation. This data should be weighed in light of the expected levels of circulating drug in clinical samples. 
Figure 1. Correlation of $\mathrm{S} / \mathrm{N}$ and titer in samples from Phase II clinical studies. $\mathrm{S} / \mathrm{N}$ strongly correlates with titer for the three (A) mAb1, (B) mAb2, and (C) mAb3 clinical studies evaluated.

$\mathrm{S} / \mathrm{N}$ : Signal-to-noise.

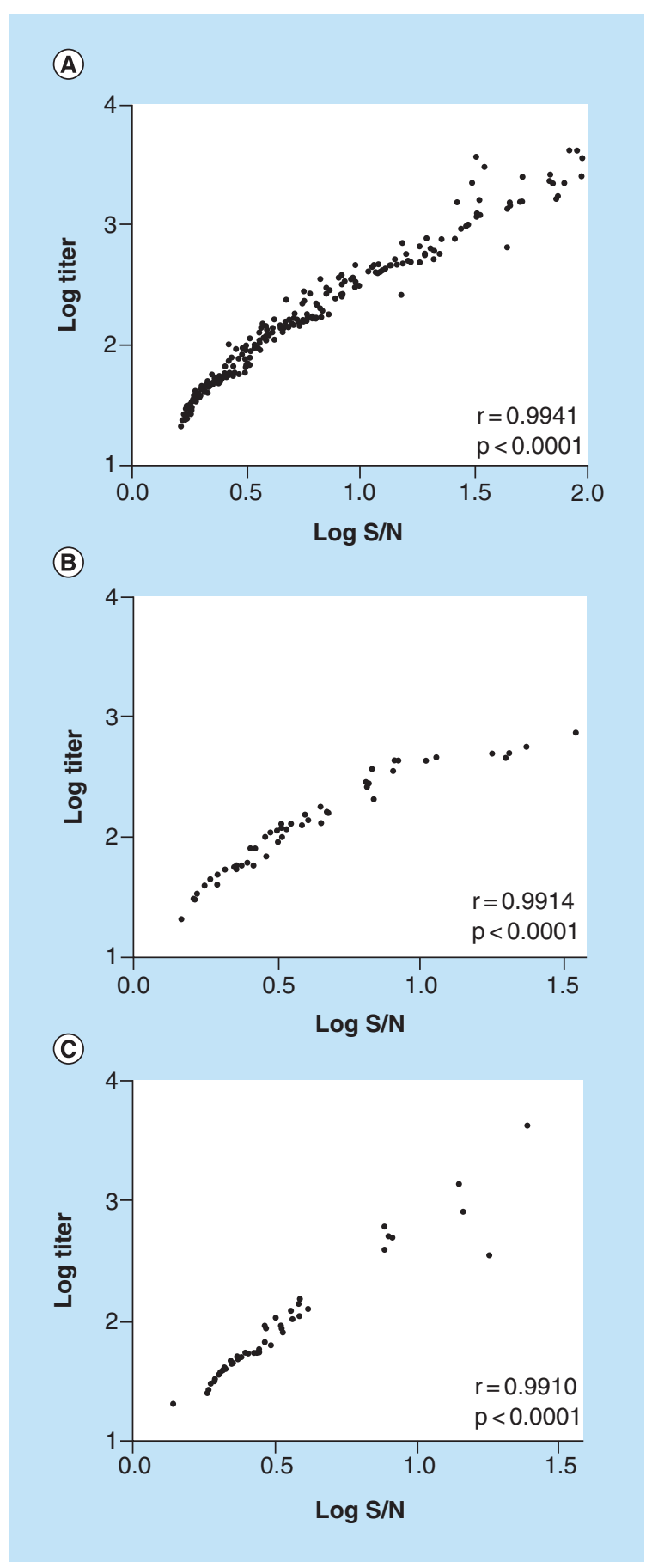

If the assay meets these criteria and anticipated drug levels are within an acceptable range, then $\mathrm{S} / \mathrm{N}$ can be used as a more efficient means to measure the magnitude of the ADA response. Use of $S / N$ involves no extra dilution steps, therefore reducing reagent and serum consumption and saving analyst time. It gives a precise value with no interpolation and may be better at differentiating between low-level ADA responses. However, excess circulating drug can disproportionately impact $\mathrm{S} / \mathrm{N}$ values relative to titer values, even at drug levels that do not impact qualitative antibody detection. Consequently, the impact of circulating drug on $\mathrm{S} / \mathrm{N}$ values should be assessed during validation, and S/N should only be used to assess magnitude when drug levels are below established thresholds. 


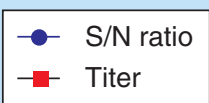

(A)
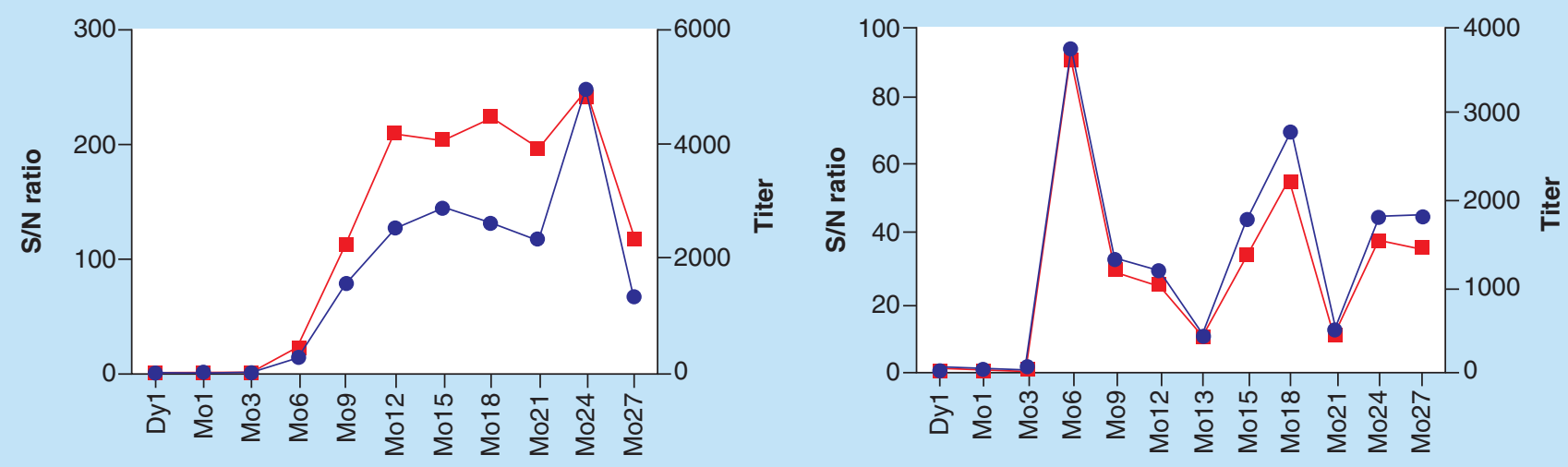

(B)
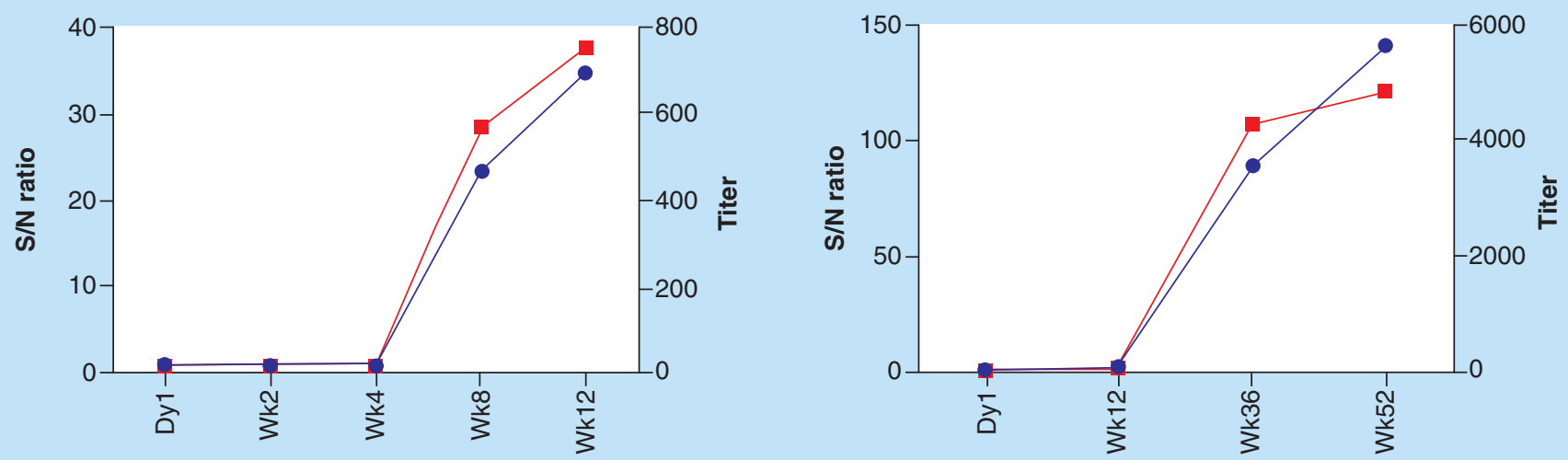

(C)
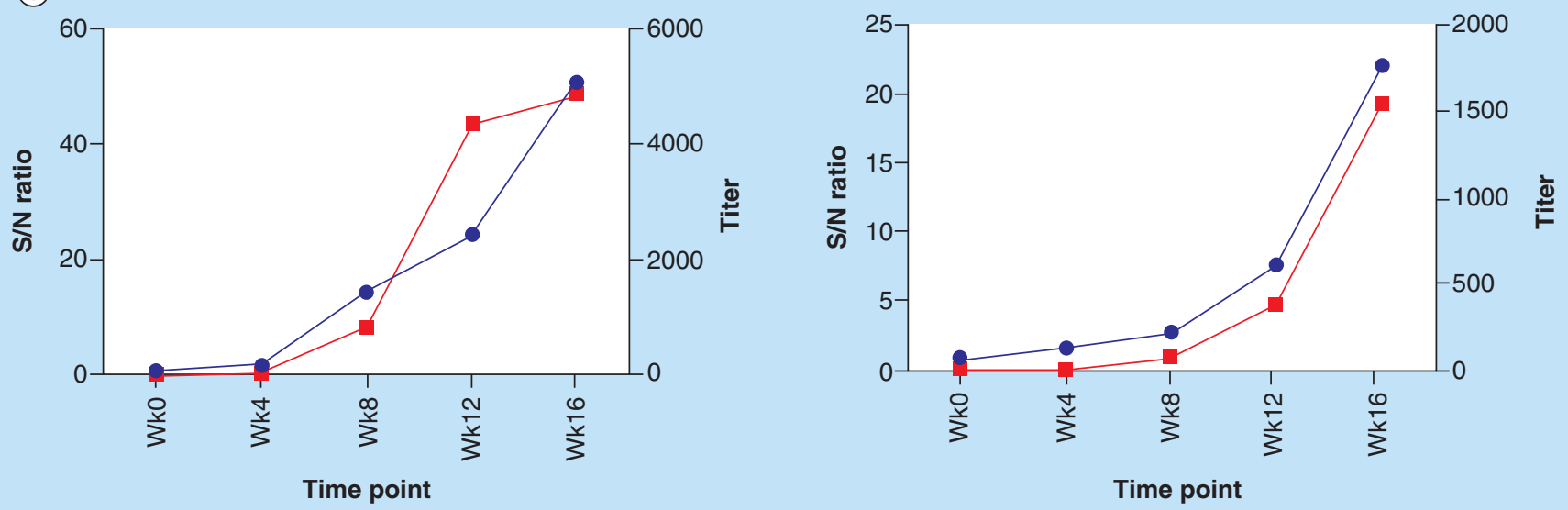

Figure 2. Individual subject profiles for subjects with the highest $\mathbf{S} / \mathbf{N}$ values. (A) mAb1, (B) mAb2 and (C) mAb3.

Dy: Day; Mo: Month; S/N: Signal-to-noise; Wk: Week.

\section{Future perspective}

Advances in ADA assay methodology have resulted in the implementation of highly sensitive and drug tolerant methods capable of detecting a wide range of antibody responses against dosed therapeutics. Differentiation of responses based on ADA magnitude can help identify those that are more likely to be clinically relevant. Due to the 
Figure 3. Impact of ADA on pharmacokinetics. Anti-mAb1 antibodies impact pharmacokinetic and this is evident when either S/N (A) or titer (B) is used. Data shown are from the highest dose group. Dashed red lines indicate the highest and lowest drug concentrations for the ADA negative samples.

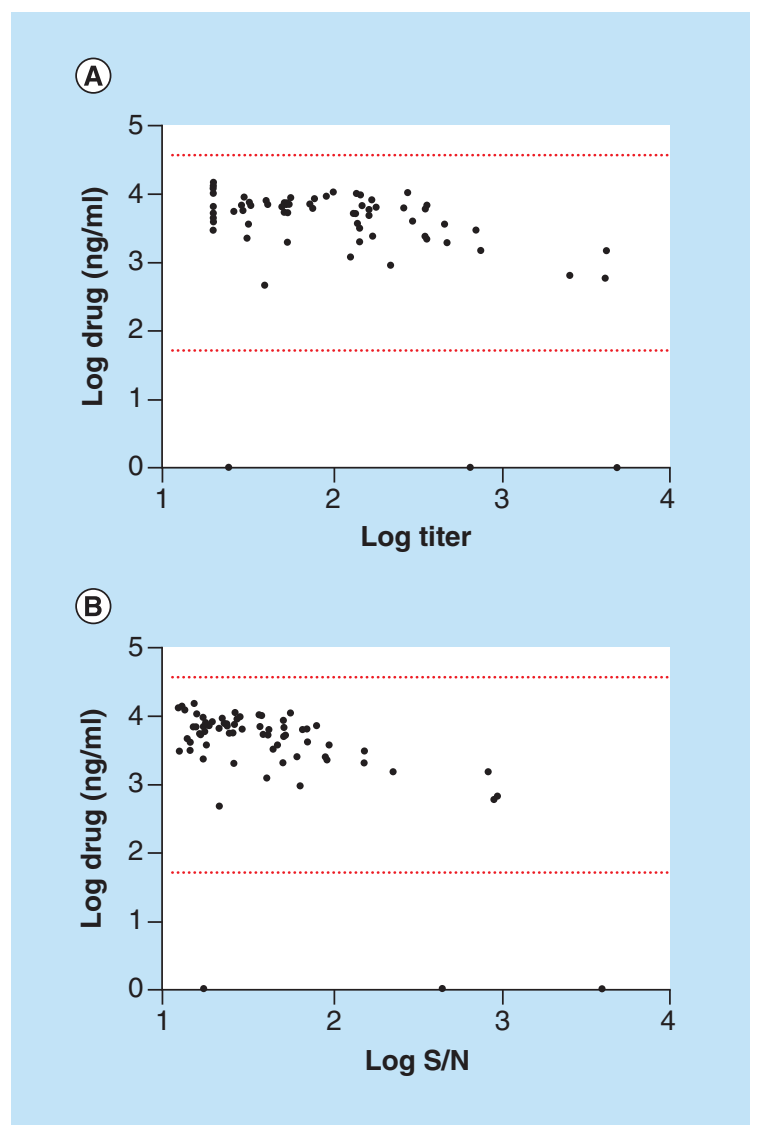
ADA: Anitdrug antibody.

resource intensive aspect of titration methods, immunogenicity monitoring in the future may increasingly include alternative measures of ADA magnitude, such as assay signal or S/N. As more sponsors start using these alternative methods, the conditions required for their successful implementation may be refined, and regulatory expectations regarding assessment of ADA magnitude will evolve.

\section{Executive summary}

- Characterization of the magnitude of an antidrug antibody (ADA) response is an important component of immunogenicity assessment.

- Measures of ADA magnitude include titer, relative antibody concentration, or assay signal or $S / N$.

- Three monoclonal antibody therapeutic ADA assays incorporating both $S / N$ and titer measures of ADA magnitude were used to monitor immunogenicity in Phase II clinical trials.

- All three assays met criteria required for successful implementation of $\mathrm{S} / \mathrm{N}$ : linearity within the range of study sample responses, inter-assay precision $\leq 20 \%$, and good recovery of $\mathrm{S} / \mathrm{N}$ in the presence of drug at concentrations observed in clinical study samples.

- $\mathrm{S} / \mathrm{N}$ correlated strongly with titer, produced similar subject ADA profiles, and generated equivalent results during analysis of impact of ADA on pharmacokinetics.

- Use of $\mathrm{S} / \mathrm{N}$ is more resource efficient, requiring less time, fewer reagents, and less sample volume than titer methods.

- $\mathrm{S} / \mathrm{N}$ can be an appropriate, quasi-quantitative measure of the magnitude of an ADA response. and $N$ Zhang for providing statistical advice, and to MB Hock, T Heath and Y Zhou for their critical review of the manuscript. 
Financial \& competing interests disclosure

Support for the study was provided by Amgen, Inc. All authors are shareholders and current or former employees of the study sponsor. $V$ Jawa is a shareholder and current employee of Merck. The authors have no other relevant affiliations or financial involvement with any organization or entity with a financial interest in or financial conflict with the subject matter or materials discussed in the manuscript apart from those disclosed.

No writing assistance was utilized in the production of this manuscript.

\section{Ethical conduct of research}

The authors state that they have obtained appropriate institutional review board approval or have followed the principles outlined in the Declaration of Helsinki for all human or animal experimental investigations. In addition, for investigations involving human subjects, informed consent has been obtained from the participants involved.

\section{Open access}

This work is licensed under the Attribution-NonCommercial-NoDerivatives 4.0 Unported License. To view a copy of this license, visit http://creativecommons.org/licenses/by-nc-nd/4.0/

\section{References}

Papers of special note have been highlighted as: $\bullet \bullet$ of considerable interest

1 Shankar G, Arkin S, Cocea L et al. Assessment and reporting of the clinical immunogenicity of therapeutic proteins and peptides-harmonized terminology and tactical recommendations. AAPS J. 16(4), 658-673 (2014).

2 Wadhwa M, Knezevic I, Kang HN, Thorpe R. Immunogenicity assessment of biotherapeutic products: an overview of assays and their utility. Biologicals 43(5), 298-306 (2015).

3 Song S, Yang L, Trepicchio WL, Wyant T. Understanding the supersensitive anti-drug antibody assay: unexpected high anti-drug antibody incidence and its clinical relevance. J. Immunol. Res. 2016, 3072586 (2016).

4 Zhou L, Hoofring SA, Wu Y et al. Stratification of antibody-positive subjects by antibody level reveals an impact of immunogenicity on pharmacokinetics. AAPS J. 15(1), 30-40 (2013).

5 EMA. Guideline on Similar Biological Medicinal Products Containing Biotechnology-Derived Proteins as Active Substance: Non-Clinical and Clinical Issues (2014). www.ema.europa.eu/docs/en_GB/document_library/Scientific_guideline/2015/01/WC500180219.pdf

6 US FDA. Scientific Considerations in Demonstrating Biosimilarity to a Reference Product (2015). www.fda.gov/downloads/drugs/guidances/ucm291128.pdf

7 Kroenke MA, Weeraratne DK, Deng $\mathrm{H}$ et al. Clinical immunogenicity of the d-amino acid peptide therapeutic etelcalcetide: method development challenges and anti-drug antibody clinical impact assessments. J. Immunol. Methods 445, 37-44 (2017).

8 Myler H, Hruska MW, Srinivasan S et al. Anti-PEG antibody bioanalysis: a clinical case study with PEG-IFN-lambda-1a and PEG-IFN-alpha2a in naive patients. Bioanalysis 7(9), 1093-1106 (2015).

9 EMA. Guideline on Immunogenicity Assessment of Biotechnology-Derived Therapeutic Proteins (2007). www.ema.europa.eu/docs/en_GB/document_library/Scientific_guideline/2009/09/WC500003946.pdf

10 US FDA. Assay Development and Validation for Immunogenicity Testing of Therapeutic Protein Products (2016; draft). www.fda.gov/downloads/Drugs/Guidances/UCM192750.pdf

-• Recent guidance providing detailed recommendations for development and validation of antidrug antibody assays.

11 Gorovits B. Antidrug antibody assay validation: industry survey results. AAPS J. 11(1), 133-138 (2009).

12 Tatarewicz SM, Mytych DT, Manning MS et al. Strategic characterization of anti-drug antibody responses for the assessment of clinical relevance and impact. Bioanalysis 6(11), 1509-1523 (2014).

13 US FDA. Guidance for Industry: Assay Development for Immunogenicity Testing of Therapeutic Proteins (2009; draft). www.fda.gov/downloads/Drugs/Guidances/UCM192750.pdf

14 Mire-Sluis AR, Barrett YC, Devanarayan V et al. Recommendations for the design and optimization of immunoassays used in the detection of host antibodies against biotechnology products. J. Immunol. Methods 289(1-2), 1-16 (2004).

15 Shankar G, Devanarayan V, Amaravadi L et al. Recommendations for the validation of immunoassays used for detection of host antibodies against biotechnology products. J. Pharm. Biomed. Anal. 48(5), 1267-1281 (2008).

-. White paper summarizing critical experimental and statistical considerations for development and validation of antidrug antibody assays. 